\title{
Effect of Custom-Molded Foot Orthoses on Foot Pain and Balance in Children With Symptomatic Flexible Flat Feet
}

\author{
Hong-Jae Lee, MD¹, Kill-Byung Lim, MD¹, JeeHyun Yoo, $\mathrm{MD}^{1}$, \\ Sung-Won Yoon, MD ${ }^{1}$, Hyun-Ju Yun, MD ${ }^{1}$, Tae-Ho Jeong, MS $^{2}$ \\ ${ }^{1}$ Department of Physical Medicine and Rehabilitation and \\ ${ }^{2}$ Institute of Sports Rehabilitation, Inje University Ilsan Paik Hospital, Goyang, Korea
}

Objective To evaluate the effect of custom-molded foot orthoses on foot pain and balance in children with symptomatic flexible flat foot 1 month and 3 months after fitting foot orthosis.

Method A total of 24 children over 6 years old with flexible flat feet and foot pain for at least 6 months were recruited for this study. Their resting calcaneal stance position and calcaneal pitch angle were measured. Individual custom-molded rigid foot orthoses were prescribed using inverted orthotic technique to control foot overpronation. Pain questionnaire was used to obtain pain sites, degree, and frequency. Balancing ability was determined using computerized posturography. These evaluations were performed prior to custom-molded foot orthoses, 1 month, and 3 months after fitting foot orthoses.

Result Of 24 children with symptomatic flexible flat feet recruited for this study, 20 completed the study. Significant $(\mathrm{p}<0.001)$ improvements in pain degree and frequency were noted after 1 and 3 months of custommolded foot orthoses. In addition, significant $(\mathrm{p}<0.05)$ improvement in balancing ability was found after 3 months of custom-molded foot orthoses.

Conclusion Short-term use of custom-molded foot orthoses significantly improved foot pain and balancing ability in children with symptomatic flexible flat foot.

Keywords Flatfoot, Foot orthoses, Pediatrics, Musculoskeletal pain, Postural balance

Received March 25, 2015; Accepted June 16, 2015

Corresponding author: Sung-Won Yoon

Department of Physical Medicine and Rehabilitation, Sports Medicine Center, Ilsan paik Hospital, 170 Juhwa-ro, Ilsanseo-gu, Goyang 10380, Korea

Tel: +82-31-910-7440, Fax: +82-31-910-7446, E-mail: 19534@paik.ac.kr

(c) This is an open-access article distributed under the terms of the Creative Commons Attribution Non-Commercial License (http://creativecommons. org/licenses/by-nc/4.0) which permits unrestricted noncommercial use, distribution, and reproduction in any medium, provided the original work is properly cited.

Copyright $\odot 2015$ by Korean Academy of Rehabilitation Medicine

\section{INTRODUCTION}

Flat feet are commonly found in children with various symptoms $[1,2]$. 'Flat feet' is a term referring to deformation of feet from hyperpronation caused by loss or immaturity of the medial longitudinal arch [3]. Flat feet are divided into pathologic flat feet with various primary causes and physiologic flat feet lacking the development of the medial longitudinal arch [4]. Flat feet in children 
are mostly physiologic flat feet. They are found in approximately $90 \%$ children under age of 2 . Development of normal longitudinal arch begins at the age of 3 to 5 years old. It is completed before the age of $10[5,6]$.

Flat feet can also be divided into flexible type and rigid type. In flexible flat feet, when the patient supports his own weight, the medial longitudinal arch will disappear. However, when the patient stands on his toes or on his heels, the medial longitudinal arch will appear. In rigid flat feet, the arch is maintained low regardless of weightbearing [7-10].

Flexible flatfeet in children can improve with age without symptoms [15]. Physiological variation of flexible flat feet does not require treatment unless it becomes symptomatic [11]. However, if physiological dysfunctions are accompanied, various symptoms may occur, including pain, reduction in exercise ability, and abnormal gait [4,12-14].

Treatment for patients suffering from symptomatic flexible flat feet include nonsurgical method and surgical method. Examples of nonsurgical methods include revision of living habits, wearing proper shoes or foot orthoses, stretching, muscle strengthening exercises, and nonsteroidal anti-inflammatory drugs. Research effort using foot orthosis nonsurgical method has been attempted to lessen foot pain. Powell et al. [16] has reported that custom-molded foot orthosis is highly effective for pediatric patients suffering from symptomatic flexible flat feet (SFFF) with symptoms of juvenile arthritis and foot pain. Although several research studies have concluded that foot orthosis could be used to control foot pain $[16,17]$, no research has been conducted to examine the changes of patient's balancing ability at the same time.

While treating patients suffering from flat feet for 15 years, we realized that children with severe flat feet had more difficulties in balancing when they stood on one leg trying to maintain their posture. In a preliminary testing for approximately 10 children with flat feet through computerized posturography to enable quantitative analysis, we verified that children with flat feet lacked balancing abilities. It has been reported that patients suffering from flat feet do lack balancing abilities while standing on one leg $[12,18]$. Therefore, the objective of this study was to comparatively analyze the frequency of pain occurrence, pain degree, changes in their ability to perform static and dynamic balancing, and functional activities of children with symptomatic flexible flat feet after wearing customized foot orthoses.

\section{MATERIALS AND METHODS}

\section{Study subjects}

We recruited pediatric patients clinically diagnosed with flat foot who visited our foot clinic between June 2012 and June 2014. Inclusion criteria were school age ( $>6$ years), complaint of foot pain four times a month or an average of at least once a week for the past 6 months, capability of independent walking and running on functional test, eversion angle of resting calcaneal stance position on both foot over $4^{\circ}$, and calcaneal pitch under $20^{\circ}$ [19]. The reason why we limited subjects less than 6 years old was because they needed to perform balanced study for purpose of this research. Exclusion criteria were bone fractures or deformities of spine or lower extremities detected on X-ray images, neurologic anomalies, infectious diseases, hemostatic disorders, malignant diseases, and current treatment for depression. Of the 24 patients selected for this study, 20 completed this study.

\section{Methods}

Biomechanical and radiological tests and the use of foot orthoses

Calcaneal pitch angle and resting calcaneal stance position (RCSP) were measured using foot X-rays and biomechanical tests. Bimanual technique was used to measure the resting calcaneal stance position. The midpoints of calcaneus were connected in the coronal plane with subjects lying at prone position. After partitioning the calcaneus in this way, the angle between the perpendicular line to the ground and the bisector of the calcaneus was measured with subjects standing to fit the ambulation angle and the ambulation base. Positive and negative values in the resting calcaneal stance position indicated inversion and eversion, respectively [20].

When a biomechanical abnormality such as overpronation was found, a customized foot orthosis was prescribed using inverted orthotic technique [21]. For example, when the initial RCSP were $-5^{\circ}$ to $-6^{\circ}$ and $-7^{\circ}$ to $-8^{\circ}$, the inverted orthotic technique prescribed $20^{\circ}$ and $25^{\circ}$ angles, respectively. For a RCSP that was $-9^{\circ}$ to $-10^{\circ}$ but lower than $-11^{\circ}$ in the severe type, $30^{\circ}$ and $35^{\circ}$ inverted orthotic technique with lateral long flanges were 

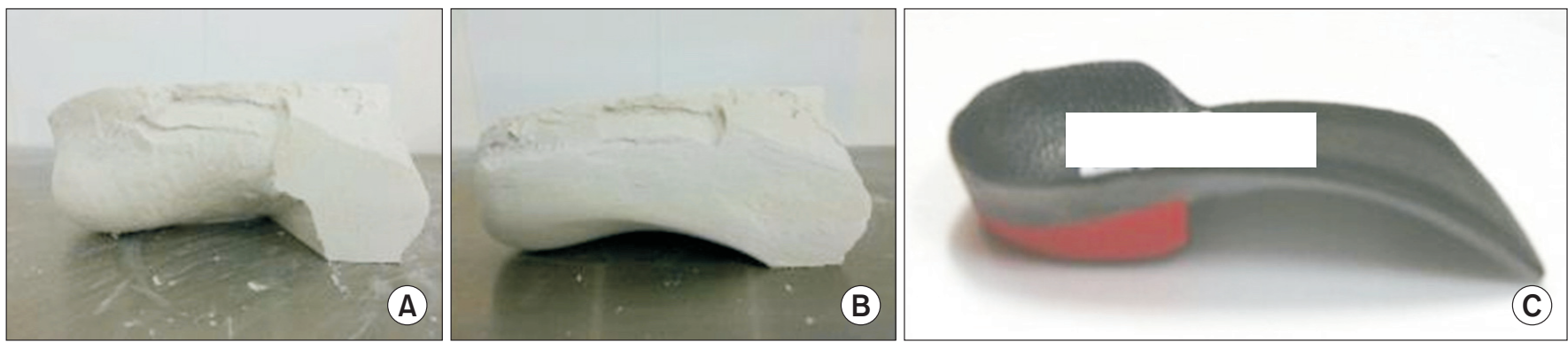

Fig. 1. Fabrication of custom molded rigid foot orthoses made with inverted orthotic technique. (A) Inverting of positive cast and platforming of the forefoot. (B) Remodeling of plantar arch from sustentaculum tali to the 1st metatarsal head. (C) Finalized orthosis.

prescribed, respectively (Fig. 1).

Fabrication of custom-modeled rigid foot orthoses was performed in the following steps. First, a negative cast was created out of the patient's foot including midpoints of calcaneous. Gypsum was then poured in the negative cast to make a positive cast of the original figure. Inverted technique was applied as prescribed by the doctor for the formerly produced cast. If $25^{\circ}$ inverted technique was applied, the first step was to adjust the midpoints of calcaneous inversion to $25^{\circ}$ on the horizontal level. A metatarsal head was then nailed to make a gap. Finally, the forefoot was filled with gypsum paste. When the anterior platform of the positive cast was hardened, a new medial calcaneal arch portion was created by adjusting the gypsum paste on the original positive cast. The new medial calcaneal arch portion will have a triangular shape. The posterior apex will be medial calcaneal tubercle point. The frontal apex will be the 1st metatarsal point. The upper apex will be the talonavicular point. On top of the newly plastered arch, thermoplastic resin was melted. Compressor cooling procedure was applied to create a new shape of the sole. Finally, a new plastic orthoses was completed by attaching additional firm and high density resin on the rear foot to prevent imbalance [20] (Fig. 1). In addition, patients and their caregivers were educated so that the children could wear the orthoses as long as possible inside their shoes for both indoor and outdoor activities.

Evaluation of pain frequency, sites, and degrees in the feet and ankles

Sites of pains in the feet, frequency of pain, and degree of pains before treatment were were compared to those at 1 month post-treatment and 3 months post-treatment.
The sites of foot pain were examined on midpoint of sole, heel base, posterior heel, Achilles tendon, and ankle articular line area. Frequency of pains was measured weekly. Visual analog scale (VAS) was used to evaluate pain frequency.

\section{Evaluation of balancing ability}

A Balance Master system (NeuroCom, Clackamas, OR, USA) was used to evaluate balancing ability, including static, dynamic, and functional test methods. Sensory limitation assessment items included static balancing ability, motor limitation assessment items for dynamic balancing ability, and functional limitation assessment items for functional balancing ability. These tests were performed before treatment with orthoses and 3 months after treatment with orthoses to compare the short-term effect. The reason why these tests were performed after wearing foot orthoses for 3 months was because children performed daily activities while continuously wearing foot orthoses. Balancing test was intended to be conducted in a real-life setting of pediatric patients wearing foot orthoses.

\section{Sensory limitation assessment for static balancing ability}

As subsections of sensory limitation assessment for this study, modified clinical test sensory interaction on balance (mCTSIB) and unilateral stance were measured. For mCTSIB, foam-interfering proprioception was placed on a force plate. Subjects stood on it and maintained their postures with eyes opened or closed for 10 seconds each for measuring the center of gravity (COG) sway velocity $(\%)$. For the unilateral stance test, subjects stood on one leg and tried to maintain their posture with their eyes opened or closed for 10 seconds on a force plate to mea- 
sure the COG sway velocity. Each test was repeated three times. Mean values were then calculated from the three repeats.

Motor limitation assessment for dynamic balancing ability For motor limitation assessment, four subsections of stability limits were tested. The limits of stability test examined how far subjects intentionally moved their COG to the left or right from the original center The reaction time, movement velocity moving the COG, endpoint and maximum excursion, and directional control were measured. Tests were repeated three times and mean values were calculated.

\section{Functional limitation assessment of functional balancing} ability

As subsections of this test, step/quick turn and tandem gait were measured. For the step/quick turn, subjects waited at a starting point, stepped onto a force plate with one leg at the examiner's starting signal, turned around $180^{\circ}$ after two steps, and returned to the starting point. During this process, turn sway $\left({ }^{\circ}\right)$ of the COG and turn time (s) were measured. For the tandem walk test, subjects walked on a test plate with toe and foot heel touching the plate to examine their walking characteristics. Step width, step speed, and end sway were measured.

\section{Statistical analyses}

Repeated-measures analysis of variance was used to compare pain degree and frequency (three time points comparison) at each time point before and after wearing foot orthoses. Wilcoxon signed-rank test was used to analyze the degree of balancing ability change (two time points comparison). SPSS ver. 21.0 (IBM SPSS, Armonk, NY, USA) was used for statistical analyses. Statistical significance was considered when $\mathrm{p}$-value was less than 0.05 .

Table 1. General characteristics of study subjects $(n=20)$

\begin{tabular}{lc}
\hline Characteristic & Value \\
\hline Sex (male:female) & $12: 8$ \\
\hline Age (yr) & $11.0 \pm 2.0$ \\
\hline Height $(\mathrm{cm})$ & $145.4 \pm 11.5$ \\
\hline Weight $(\mathrm{kg})$ & $40.3 \pm 8.2$ \\
\hline
\end{tabular}

Values are presented as number or mean \pm standard deviation.

\section{RESULTS}

\section{Subject general characteristics}

Of the 20 patients who completed this study, the boy to girl ratio was 12 to 8 . Their average age, height, and weight were $11.0 \pm 2.05$ years, $145.40 \pm 11.53 \mathrm{~cm}$, and $40.28 \pm 8.16 \mathrm{~kg}$, respectively (Table 1 ).

\section{Biomechanical and radiological test findings}

The average RCSP of all participants was $15.2^{\circ}$ on the left and $15.3^{\circ}$ on the right (Table 2). There were 10 patients who had severe case of pitch angle (under $15^{\circ}$ ). The average calcaneal pitch angle of the 20 patients was $-7.25^{\circ}$ on the left and $-6.05^{\circ}$ on the right on biomechanical test.

\section{Change in pain regions, degrees, and frequencies}

Pain sites answered by the subjects included middle sole $(\mathrm{n}=14)$, platar heel $(\mathrm{n}=5)$, Achilles tendon and posterior heel $(n=6)$, and anterior ankle joint line $(n=4)$. Nine of the 20 children complained of pain in more than two regions (Table 3 ).

Compared to pain frequency at $15.65 \pm 10.39$ per month before treatment, pain frequency was significantly decreased to $9.23 \pm 9.05$ per month at 1 month after treatment and $6.88 \pm 8.18$ per month at 3 months after treatment. Since the frequency of pain occurrence was statistically significant $(\mathrm{p}<0.001)$ over time, pain frequency showed a linear pattern. Statistically significant differences were evident between before treatment and 1 month after treatment $(\mathrm{p}=0.007)$ or 3 months after treat-

Table 2. Resting calcaneal stance position (RCSP) and calcaneal pitch angle

\begin{tabular}{lrr}
\hline & Right & Left \\
\hline RCSP & $-6.05 \pm 3.19$ & $-7.25 \pm 3.69$ \\
Calcaneal pitch angle & $15.2 \pm 5.47$ & $15.3 \pm 5.80$ \\
\hline
\end{tabular}

Values are presented as mean \pm standard deviation.

Table 3. Location of pain in the foot

\begin{tabular}{|lc|}
\hline \multicolumn{1}{|c}{ Location of pain } & No. \\
\hline Middle sole $(\operatorname{arch})$ & 14 \\
\hline Plantar heel & 5 \\
\hline Posterior heel \& Achilles tendon & 6 \\
\hline Ankle joint line & 4 \\
\hline
\end{tabular}

Patients can answer multiple pain sites. 


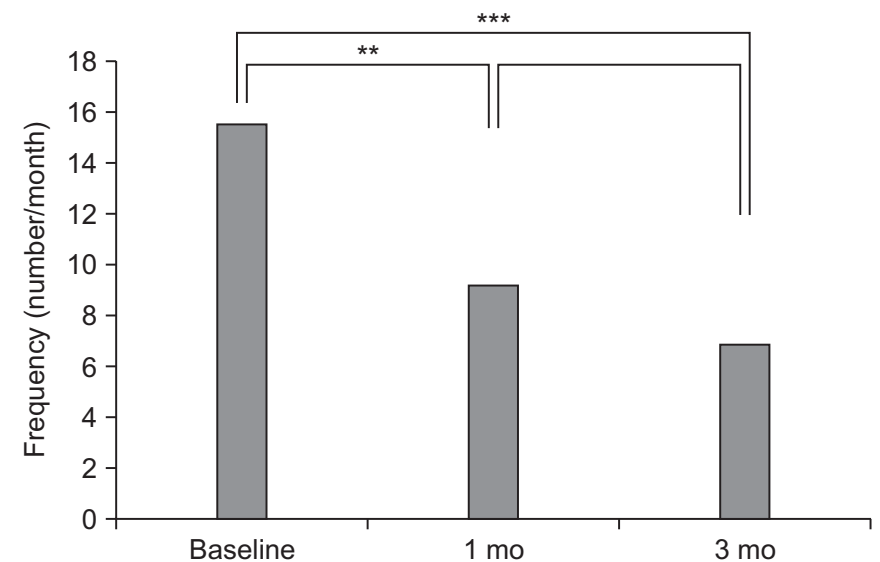

Fig. 2. Frequency of pain occurrence. ${ }^{* *} \mathrm{p}<0.01,{ }^{* * *} \mathrm{p}<0.001$ of using repeated-measures analysis of variance.

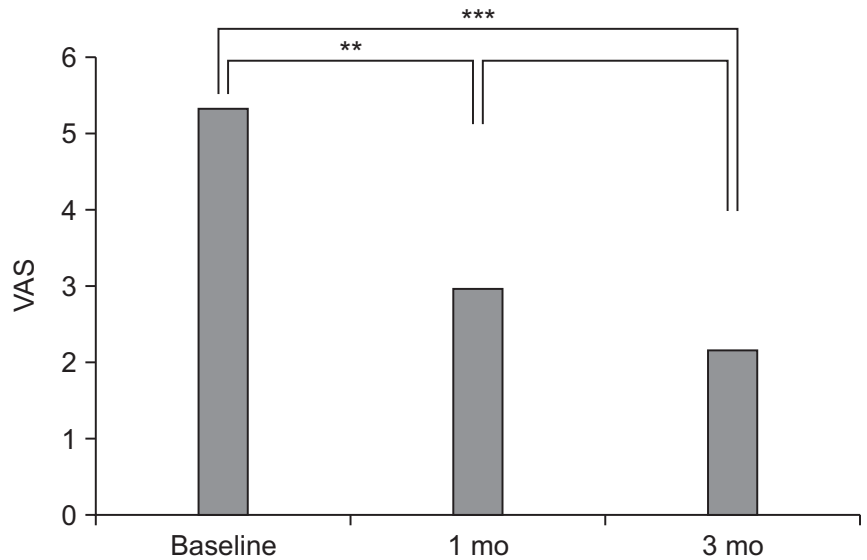

Fig. 3. Visual analog scale (VAS) in the main pain site. ${ }^{* *} \mathrm{p}<0.01,{ }^{* * *} \mathrm{p}<0.001$ of using repeated-measures analysis of variance.

Table 4. Comparison of static, dynamic, and functional balancing ability after fitting foot orthoses

\begin{tabular}{|lccc}
\hline & Before & $\mathbf{3 ~ m o}$ & p-value \\
\hline Static balance parameters & & & \\
\hline Unilateral stance-COG sway velocity $(\%)$ & & & \\
\hline Left EO & $1.67 \pm 1.31$ & $1.36 \pm 0.99$ & 0.210 \\
\hline Right EO & $1.27 \pm 0.70$ & $1.43 \pm 0.67$ & 0.203 \\
\hline Left EC & $3.18 \pm 1.34$ & $2.85 \pm 1.47$ & 0.398 \\
\hline Right EC & $3.12 \pm 1.27$ & $2.51 \pm 1.42$ & $0.006^{*}$ \\
\hline Dynamic balance parameters & & & \\
\hline Movement velocity $(\% / s)$ & & & 0.218 \\
\hline Forward & $6.26 \pm 1.85$ & $7.03 \pm 2.57$ & 0.150 \\
\hline Back & $4.27 \pm 1.36$ & $4.99 \pm 1.83$ & 0.322 \\
\hline Right & $6.80 \pm 2.73$ & $7.99 \pm 3.46$ & $0.002^{*}$ \\
\hline Left & $6.72 \pm 1.96$ & $8.93 \pm 2.59$ & 0.526 \\
\hline Endpoint $(\%)$ & & & 0.411 \\
\hline Forward & $81.85 \pm 16.47$ & $86.45 \pm 23.54$ & 0.679 \\
\hline Back & $66.90 \pm 16.43$ & $71.35 \pm 19.21$ & $0.046^{*}$ \\
\hline Right & $91.35 \pm 22.30$ & $94.90 \pm 18.98$ & \\
\hline Left & $87.00 \pm 21.71$ & $102.65 \pm 19.44$ & \\
\hline Functional balance parameters & & & \\
\hline Step/quick turn & & & $0.020^{*}$ \\
\hline Turn time - right $(\mathrm{s})$ & & & $0.040^{*}$ \\
\hline Turn time - left $(\mathrm{s})$ & $1.07 \pm 0.67$ & $0.85 \pm 0.54$ & $0.017^{*}$ \\
\hline Turn sway - right $\left({ }^{\circ} / \mathrm{s}\right)$ & $1.16 \pm 0.98$ & $0.91 \pm 0.53$ & 0.985 \\
\hline Turn sway - left $(\% / s)$ & $30.04 \pm 9.64$ & $25.97 \pm 7.26$ & $26.61 \pm 7.25$ \\
\hline
\end{tabular}

Values are presented as mean \pm standard deviation.

COG, center of gravity; EO, eye open; EC, eye closed.

${ }^{*} \mathrm{p}<0.05$ by Wilcoxon signed-rank test. 
ment $(\mathrm{p}<0.001)$ in postexperimnetal analysis. However, the comparison between 1 month and 3 months after treatment was statistically insignificant $(\mathrm{p}=0.075)$. These findings indicated that the frequency of pain was improved within 1 month after wearing foot orthoses (Fig. 2).

The degree of pain in the most complained sites was evaluated using VAS. Compared to the degree of pain at $5.30 \pm 2.24$ before trement, the degree of pain was significantly reduced to $2.95 \pm 2.22$ at 1 month after treatment and $2.15 \pm 2.33$ at 3 months after treatment. Since pain degrees showed statistically significant $(\mathrm{p}<0.001)$ difference over time, they followed a linear pattern. Significant differences were evident in comparisons between before treatment and 1 month after treatment $(p=0.01)$ or between before treatment and 3 months after treatment $(\mathrm{p}<0.001)$. However, the degrees of pain at 1 month after treatment and at 3 months after treatment were not significantly $(\mathrm{p}=0.085)$ different. Thereofre, the degree of pain in the most affected sites could be significantly decreased in 1 month after after wearing foot orthoses (Fig. 3).

\section{Changes in balancing ability}

\section{Static balance}

In a unilateral stance test with eyes opened, when the COG sway velocity before treatment was compared to that at 3 months after treatment, there was no statistically significant improvement. When the eyes were closed, the velocity of the right was decreased from $3.12 \pm 1.27(\% / \mathrm{s})$ to $2.51 \pm 1.42(\%)$, showing statistically significant improvements in static balance (Table 4).

\section{Dynamic balance}

The limit of stability subsections were measured with the child moving in four directions. In the movement velocity domain, left movement speed was improved significantly from $6.72 \pm 1.96(\% / \mathrm{s})$ to $8.93 \pm 2.59(\%)$. However, the forward, backward, and rightward movements showed no statistically significant improvement. In addition, the endpoint excursion area performed to the left showed a statistically significant improvement (from $87.00 \% \pm 21.71 \%$ to $102.65 \% \pm 19.44 \%$ ) (Table 4 ).

\section{Functional balance}

The turn time to the right in step/quick turn showed a statistically significant improvement from $1.07 \pm 0.67$ (s) to $0.85 \pm 0.54$ (s). Similary, the turn time to the left in step/ quick turn was also significantly improved from $1.16 \pm 0.98$ (s) to $0.91 \pm 0.53$ (s) did. In the turn sway test, statistically significant decrease was found in the left side from $28.58 \pm 13.54(\%$ s) to $26.61 \pm 7.25(\%$ s) (Table 4$)$.

\section{DISCUSSION}

\section{Discussion on the decrease in foot pain}

In the present study, the frequency of pain complaints and pain intensity were assessed over 3 months after children with SFFF wore foot orthoses. A large and statistically significant decrease in pain was found. In particular, the improvement in pain was found to be the largest during the first month.

Foot orthoses have been previously used to treat flat feet with foot pain $[16,17]$. In a recent Cochrane review, it has been suggested that the use of foot orthoses in children with SFFF has limited evidence [22,23]. In a randomized control study in children under 6 years old with flat feet, Wegner has compared a control group, a corrective orthopedic shoe group, a Helfet heel-cup group, and a custom-molded plastic insert group for 3 years and reported a similar improvement in all 4 groups based on radiographic findings [24]. Whitford and Esterman [17] have compared a custom-made foot orthosis with a ready-made orthosis and reported that there is improvement in pain and motor function after 1 year of wearing the orthosis without statistically significant difference between the two groups. Therefore, there is still a lot of debate regarding the effect of foot orthoses on SFFF.

In this study, after children over 6 years old with SFFF and complaints of repeated pain wore foot orthoses for 3 months, there was a large and statistically significant $(\mathrm{p}<0.001)$ reduction in pain intensity and pain frequency. The largest improvement was seen after wearing foot orthoses for one month. The main cause of this change was concluded to be wearing custom-made foot orthoses using inverted orthotic technique. Because these chronic and recurring pains persisting for over 6 months were from children over 6 years old, a natural improvement in foot pain within one month would not be expected. Therefore, the reduction in foot pain was likely to be due to foot orthoses.

After biomechanical analysis of patient's foot and ankle, the inverted orthotic technique was applied, in which the position of the calcaneus was moved vertically. This tech- 
nique could have prevented overpronation of the foot.

The mechanisms that can cause foot pain in SFFF include overuse injury [25] and referred pain due to myofascial pain syndrome in tibialis posterior or gastrocnemius [26]. The following mechanisms can cause pain in the sole of the foot (the arch and the inferior aspect of the heel). Flexible flat feet in children have high mechanical instability. Therefore, the intrinsic muscles of the feet have to be highly active to maintain the balance of the body. Therefore, there will be a high risk of muscle fatigue and pain due to overuse. There is also a high risk of muscle pain and fatigue due to overuse of tibialis posterior, the primary muscle that maintains the arch of the foot and restricts overpronation. Because tibialis posterior is a deep muscle, it often causes referred pain that can be expressed in the arch and the inferior aspect of the heel [26].

Leung et al. [27] have performed gait analysis before and after wearing foot orthoses and reported that orthoses could reduce the extent and duration of abnormal pronation during the stance phase. This could potentially reduce strain on the plantar ligaments. Therefore, foot orthoses might also reduce plantar foot pain by reducing overpronation and alleviating the stress on the plantar ligaments.

The mechanisms that can cause pain in the posterior part of the heel (the posterior aspect of calcaneus and the Achilles tendon) are as follows. From a mechanical perspective, in flexible flat feet, the midtarsal joint does not undergo resupination during the push-off phase. Thereofre, the midfoot is in an unlocked state, which makes push off inefficient. In order to compensate for the problem of inefficient push off, triceps surae muscle will contract with a little more force. When there is repeated and excessive contraction of triceps surae muscle, increased stress will be added to the Achilles tendon and the tendon insertion site on calcaneus. This will cause pain in these areas.

Inverted orthotic technique was used to manufacture custom-made foot orthoses for those children with flexible flat feet. This prevented abnormal overpronation, which reduced stress due to excessive contraction of the intrinsic and extrinsic muscles maintaining the arch of the foot. Therefore, the pain on the plantar aspect and the posterior heel was reduced.
Discussion on the improvements in posture maintenance and balance ability

In the assessment for static balance ability, there was a statistically significant reduction in the COG sway velocity in a unilateral stance with the right eye closed. In the assessment of dynamic balance ability, there was a statistically significant improvement in movement velocity and endpoint of first movement on the left side. In the assessment of functional balance ability during step/quick turn, there was a statistically significant improvement in turn time in both directions and turn sway in the left direction (Table 4).

The present study aimed to investigate whether posture maintenance and balance ability could be improved after providing biomechanical stability to the foot and lower limb using foot orthoses manufactured with the inverted orthotic technique.

In the assessment of static balance ability (posture maintenance ability), when measuring sway speed was in a unilateral stance, there was a significant decrease in sway with eyes closed. Maintaining a unilateral stance with eyes closed requires the use of only proprioceptive information from the foot and ankle as well as tactile senses of the sole without any visual information. The fact that the balance ability was improved under these conditions after wearing the foot orthoses indicated that the foot orthoses provided more proprioceptive stimulation from the foot and ankle joints and more tactile stimulation to the plantar aspect or that they provided better quality information. Feuerbach et al. [28] have studied dermal touch stimulation and the ability to sense joint position by applying an ankle orthoses and found that the ability to sense joint position is improved due to an increase in the afferent feedback from receptors in the skin of the foot and ankle. In fact, foot orthoses were made using the inverted orthotic technique to place the subtalar joint closer to a neutral position and place the midtarsal joint closer to the normal position. By restoring the positions of joints in this way, the orthoses might have improved balance ability because they promoted the transmission of normal afferent signals from the proprioceptors in the ankle and the skin receptors in the plantar aspect of the foot.

Another reason for the improvement in posture maintenance ability was that the foot orthoses used in the present study were able to provide mechanical stability 
to the foot. Due to the nature of flexible flat feet in children, there is a large amount of mechanical instability in the bones of the feet. However, custom-made plastic foot orthoses can raise and support the whole arch of the foot. Therefroe, they can greatly reduce mechanical instability in the bones of the foot.

In the assessment of dynamic balance ability, there was a significant improvement in movement speed and endpoint distance of the first movement in the left direction. The movement speed and endpoint of first movement in the right direction also showed an improvement following treatment, although there as no significant improvement. This assessment required the subjects to dynamically shift their COG according to instructions on a computer screen. The fact that an improvement was seen in this assessment indicated that that the subjects were able to voluntarily move their bodies. This was made possible because subjects acquired mechanical stability in their feet with more precise afferent signals relating to joint position from the feet and ankles.

In the step/quick turn test to assess functional balance ability, subjects showed statistically significant improvement in turn time in both directions and in turn sway in the left direction. For this test, the subjects started to step forward with one foot, turned $180^{\circ}$ after a total of two forward steps, and then returned to the starting point. The sway in COG during turning and the turn time were measured. Given that the feet were responsible for balance during turning, the significant improvement in this test indicated that postural stability was increased as a result of wearing the foot orthoses. This was made possible only when mechanical stability was provided to the feet and precise afferent signals could be transmitted from the feet and ankles.

In summary, foot orthoses were made using inverted orthotic technique for children with flexible flat feet accompanied by foot pain. After wearing orthoses, these children not only showed a considerable reduction in foot pain frequency and pain intensity, but also showed improvement in static, dynamic, and functional balance ability.

This study had several limitations. First, it was a smallscale study with only 20 subjects. In addition, age restrictions had to be enforced in order to measure balance ability. If changes in pain had been assessed without an age restriction, it would have been possible to perform a larger scale study including younger children. Another limitation was that the follow-up period was relatively short at 3 months. Moreover, changes in balance ability after 3 months without wearing the foot orthoses could not be assessed. Therefore, it is unclear whether balance ability without the orthoses can be improved after 3 months. With a short-term 3-month study, it is difficult to provide guidelines for the duration that children should wear foot orthoses to have even better effect. Hence, subsequent studies are needed to determine the carryover effect after children have worn the foot orthoses for 1 year and assess their balance ability after wearing or not wearing the orthoses. In addition, this study did not take into account body habitus or body mass index of children. However, of our study subjects, none was visibly obese. Additional studies will be required to overcome these limitations.

In this study, we recruited children with flexible flat feet and foot pain. When they wore customized foot orthoses produced with inverted orthotic technique, pain frequency and degree were significantly improved. Static, dynamic, and functional balancing abilities were also improved in 3 months. Therefore, custom molded foot orthoses made with inverted orthotic technique could be effective for pediatric patients with symptomatic flexible flat feet.

\section{CONFLICT OF INTEREST}

No potential conflict of interest relevant to this article was reported.

\section{REFERENCES}

1. Sullivan JA. Pediatric flatfoot: evaluation and management. J Am Acad Orthop Surg 1999;7:44-53.

2. Garcia-Rodriguez A, Martin-Jimenez F, Carnero-Varo M, Gomez-Gracia E, Gomez-Aracena J, FernandezCrehuet J. Flexible flat feet in children: a real problem? Pediatrics 1999;103:e84.

3. Kim SB, Yoon K, Park HS, Kwak H, Ha NJ, Park JS. Radiologic measurement of flatfoot. J Korean Acad Rehabil Med 2000;24:995-1001.

4. Harris EJ, Vanore JV, Thomas JL, Kravitz SR, Mendelson SA, Mendicino RW, et al. Diagnosis and treatment of pediatric flatfoot. J Foot Ankle Surg 2004;43:341-73. 
5. Staheli LT, Chew DE, Corbett M. The longitudinal arch: a survey of eight hundred and eighty-two feet in normal children and adults. J Bone Joint Surg Am 1987;69:426-8.

6. Mosca VS. Flexible flatfoot in children and adolescents. J Child Orthop 2010;4:107-21.

7. Fabry G. Clinical practice. Static, axial, and rotational deformities of the lower extremities in children. Eur J Pediatr 2010;169:529-34.

8. Yagerman SE, Cross MB, Positano R, Doyle SM. Evaluation and treatment of symptomatic pes planus. Curr Opin Pediatr 2011;23:60-7.

9. Nemeth B. The diagnosis and management of common childhood orthopedic disorders. Curr Probl Pediatr Adolesc Health Care 2011;41:2-28.

10. Houghton KM. Review for the generalist: evaluation of pediatric foot and ankle pain. Pediatr Rheumatol Online J 2008;6:6.

11. Yan GS, Yang Z, Lu M, Zhang JL, Zhu ZH, Guo Y. Relationship between symptoms and weight-bearing radiographic parameters of idiopathic flexible flatfoot in children. Chin Med J (Engl) 2013;126:2029-33.

12. Lin CJ, Lai KA, Kuan TS, Chou YL. Correlating factors and clinical significance of flexible flatfoot in preschool children. J Pediatr Orthop 2001;21:378-82.

13. Evans AM, Rome K, Peet L. The foot posture index, ankle lunge test, Beighton scale and the lower limb assessment score in healthy children: a reliability study. J Foot Ankle Res 2012;5:1.

14. Krul M, van der Wouden JC, Schellevis FG, van Suijlekom-Smit LW, Koes BW. Foot problems in children presented to the family physician: a comparison between 1987 and 2001. Fam Pract 2009;26:174-9.

15. Pfeiffer M, Kotz R, Ledl T, Hauser G, Sluga M. Prevalence of flat foot in preschool-aged children. Pediatrics 2006;118:634-9.

16. Powell M, Seid M, Szer IS. Efficacy of custom foot orthotics in improving pain and functional status in children with juvenile idiopathic arthritis: a randomized trial. J Rheumatol 2005;32:943-50.
17. Whitford D, Esterman A. A randomized controlled trial of two types of in-shoe orthoses in children with flexible excess pronation of the feet. Foot Ankle Int 2007;28:715-23.

18. Benedetti MG, Ceccarelli F, Berti L, Luciani D, Catani F, Boschi M, et al. Diagnosis of flexible flatfoot in children: a systematic clinical approach. Orthopedics 2011;34:94.

19. Bok SK, Kim BO, Lim JH, Ahn SY. Effects of custommade rigid foot orthosis on pes planus in children over 6 years old. Ann Rehabil Med 2014;38:369-75.

20. Valmassy RL. Clinical biomechanics of the lower extremities. 1st ed. New York: Mosby; 1996. p. 243-77.

21. Blake RL. Inverted functional orthosis. J Am Podiatr Med Assoc 1986;76:275-6.

22. Rome K, Ashford RL, Evans A. Non-surgical interventions for paediatric pes planus. Cochrane Database Syst Rev 2010;(7):CD006311.

23. Evans AM, Rome K. A Cochrane review of the evidence for non-surgical interventions for flexible pediatric flat feet. Eur J Phys Rehabil Med 2011;47:69-89.

24. Wenger DR, Mauldin D, Speck G, Morgan D, Lieber RL. Corrective shoes and inserts as treatment for flexible flatfoot in infants and children. J Bone Joint Surg Am 1989;71:800-10.

25. Lorimer D, French G, O'Donnell M, Burrow JG. Neale's disorders of the foot: diagnosis and management. 6th ed. Edinburgh: Churchill Livingstone; 2002. p. 306-7.

26. Travell JG, Simons DG. Travell \& Simons' myofascial pain and dysfunction: the trigger point manual. Baltimore: Williams \& Wilkins; 1992. p. 460-1.

27. Leung AK, Mak AF, Evans JH. Biomedical gait evaluation of the immediate effect of orthotic treatment for flexible flat foot. Prosthet Orthot Int 1998;22:25-34.

28. Feuerbach JW, Grabiner MD, Koh TJ, Weiker GG. Effect of an ankle orthosis and ankle ligament anesthesia on ankle joint proprioception. Am J Sports Med 1994;22:223-9. 